\title{
Computer-Aided Detection with Automated Breast Ultrasonography for Suspicious Lesions Detected on Breast MRI
}

\author{
Sanghee Kim, Bong Joo Kang, Sung Hun Kim, Jeongmin Lee, Ga Eun Park \\ Department of Radiology, Seoul St. Mary's Hospital, The Catholic University of Korea, Seoul, Korea
}

\section{Original Article}

Received: January 13, 2019

Revised: January 24, 2019

Accepted: January 25, 2019

\section{Correspondence to:}

Bong Joo Kang, M.D., Ph.D. Department of Radiology, Seoul St. Mary's Hospital, College of Medicine, The Catholic University of Korea, 222, Banpo-daero, Seocho-gu, Seoul 06591, Korea. Tel. +82-2-2258-6253

Fax. +82-2-599-6771

E-mail: gmlionmain@gmail.com

This is an Open Access article distributed under the terms of the Creative Commons Attribution Non-Commercial License (http://creativecommons.org/licenses/ by-nc/4.0/) which permits unrestricted non-commercial use, distribution, and reproduction in any medium, provided the original work is properly cited.

\section{INTRODUCTION}

Automated breast ultrasonography (ABUS) has been developed in recent decades and proposed as a promising tool for overcoming the disadvantages of hand-held

ultrasonography (HHUS) (1). Although HHUS technology has progressed and is still the

Purpose: The aim of this study was to evaluate the diagnostic performance of a computer-aided detection (CAD) system used with automated breast ultrasonography (ABUS) for suspicious lesions detected on breast MRI, and CAD-false lesions.

Materials and Methods: We included a total of 40 patients diagnosed with breast cancer who underwent ABUS (ACUSON S2000) to evaluate multiple suspicious lesions found on MRI. We used CAD (OVCAD $\left.{ }^{\mathrm{TM}}\right)$ in all the ABUS examinations. We evaluated the diagnostic accuracy of CAD and analyzed the characteristics of CAD-detected lesions and the factors underlying false-positive and false-negative cases. We also analyzed false-positive lesions with CAD on ABUS.

Results: Of a total of 122 suspicious lesions detected on MRI in 40 patients, we excluded 51 daughter nodules near the main breast cancer within the same quadrant and included 71 lesions. We also analyzed 23 false-positive lesions using CAD with ABUS. The sensitivity, specificity, positive predictive value, and negative predictive value of CAD (for 94 lesions) with ABUS were 75.5\%, 44.4\%, 59.7\%, and 62.5\%, respectively. $C A D$ facilitated the detection of $81.4 \%$ (35/43) of the invasive ductal cancer and $84.9 \%(28 / 33)$ of the invasive ductal cancer that showed a mass (excluding non-mass). CAD also revealed $90.3 \%$ (28/31) of the invasive ductal cancers measuring larger than $1 \mathrm{~cm}$ (excluding non-mass and those less than $1 \mathrm{~cm}$ ). The mean sizes of the true-positive versus false-negative mass lesions were $2.08 \pm 0.85 \mathrm{~cm}$ versus 1.6 $\pm 1.28 \mathrm{~cm}(P<0.05)$. False-positive lesions included sclerosing adenosis and usual ductal hyperplasia. In a total of 23 false cases of CAD, the most common (18/23) cause was marginal or subareolar shadowing, followed by three simple cysts, a hematoma, and a skin wart.

Conclusion: $C A D$ with ABUS showed promising sensitivity for the detection of invasive ductal cancer showing masses larger than $1 \mathrm{~cm}$ on MRI.

Keywords: Breast cancer; Magnetic resonance imaging; Computer-aided detection; Automated breast ultrasonography 
standard breast US method, it is operator-dependent and nonreproducible, and cannot display breast lesions in the coronal plane $(2,3)$.

ABUS was primarily developed as a screening tool. Unlike HHUS, it is less operator-dependent and can acquire full three-dimensional breast US volumes that are reproducible any time. To provide complete coverage of both breasts, ABUS consists of two to five acquisitions per breast, with each acquisition comprising over 300 transverse and reconstructed images in the coronal and sagittal planes. Consequently, the large ABUS volumes might induce oversight errors involving specific malignancies. Accurate computer-aided detection (CAD) software may facilitate the detection of cancers in ABUS data sets and enhance the efficiency of radiologist work flows (4).

Research using CAD for ABUS is not exhaustive, with few studies using CAD with ABUS for suspicious lesions detected on breast MRI.

The purpose of this study was to evaluate the diagnostic performance of CAD with ABUS for suspicious lesions detected on breast MRI and to analyze the nature of CADdetected lesions and determine the factors triggering false readings.

\section{MATERIALS AND METHODS}

\section{Patient Population}

The Institutional Review Board approved the study. All patients provided informed consent for the study. Between January 1 and December 31, 2017, we prospectively enrolled 40 women (age range, 30-76 years; mean age, 50.4 \pm 9.8 years) who underwent HHUS and ABUS for multiple suspicious lesions detected on preoperative MRI. The 40 patients carried a total of 122 suspicious lesions under categories 4, 5, and 6 based on the American College of Radiology Breast Imaging Reporting and Data System (ACR BI-RADS). Most lesions were ultimately diagnosed using ultrasound-guided needle biopsy or surgical results.

Of the $122 \mathrm{MRI}$-detected lesions, we excluded 51 daughter nodules located near the main breast cancer within the same quadrant, ultimately including 71 lesions: 49 identified as malignant, 15 as nonmalignant, and 7 lesions with no change or that disappeared on followup MRI. Among the 64 pathologically confirmed lesions, 58 were confirmed via ultrasound-guided biopsy, 4 via mammography-guided biopsy, 1 was confirmed via ultrasound-guided localization prior to surgery, and 1 was confirmed via MRI-guided biopsy. The 49 lesions that were identified as malignant lesions included 43 cases of invasive ductal carcinoma (IDC), 2 involving ductal carcinoma in situ (DCIS), 3 mucinous carcinomas, and 1 case of invasive lobular carcinoma. Among the 15 nonmalignant lesions, including benign and borderline lesions, there were six cases of fibrocystic change, two of sclerosing adenosis, two of intraductal papilloma, 1 of fibroadenoma, 1 of stromal fibrosis, 1 of intramammary lymph node, 1 of ductal hyperplasia, and 1 of atypical ductal hyperplasia. We also analyzed 23 lesions showing false $C A D$ readings: marginal or subareolar shadows manifesting as pseudo-lesions (18/23), typical benign cysts (3/23), a hematoma (1/23), and a skin wart (1/23). A final total of 94 lesions including 71 MRI-detected suspicious lesions and 23 pseudo-lesions with false CAD readings were analyzed (Table 1 ).

\section{Breast MRI}

All patients underwent MR imaging of the breast at our institution, performed with a 3T MR imaging system (Ingenia, Philips, Eindhoven, The Netherlands and Verio, Siemens Medical Solutions, Erlangen, Germany). Images of both breasts were obtained in the axial plane with the patient lying in the prone position. In this study, the following sequences were used: axial, turbo spin-echo T2weighted imaging (repetition time [TR]/echo time [TE] $4530 / 93 \mathrm{~ms}$, flip angle $80^{\circ}, 34$ slices with field of view [FOV] $320 \mathrm{~mm}$, matrix $576 \times 403$, 1 number of excitations,

Table 1. Characteristics of Patients and Lesions

\begin{tabular}{lc}
\hline Total & 94 \\
\hline Age & \\
\hline Mean \pm SD & $50.4 \pm 9.8$ \\
\hline Median (Range) & $49(30-72)$ \\
\hline Pathology & 71 \\
\hline Benign & $11(11.7 \%)$ \\
\hline Borderline & $4(4.3 \%)$ \\
\hline Malignancy & $49(52.1 \%)$ \\
\hline No change/disappeared on follow-up & $7(7.4 \%)$ \\
\hline CAD marker for pseudo-lesion & 23 \\
\hline Marginal or subareolar shadowing & $18(19.1 \%)$ \\
\hline Cyst & $3(3.2 \%)$ \\
\hline Hematoma & $1(1.1 \%)$ \\
\hline Skin wart & $1(1.1 \%)$ \\
\hline
\end{tabular}

$C A D=$ computer-aided detection; $S D=$ standard deviation 
$4 \mathrm{~mm}$ slice thickness, acquisition time 2 minutes 28 second). Pre- and post-contrast, axial, T1-weighted flash three-dimensional (3D), volumetric interpolated breathhold examination (TR/TE 4.4/1.7 ms, flip angle $10^{\circ}, 1.2 \mathrm{~mm}$ slice thickness with no gap, and acquisition time $60 \mathrm{~s}$ ). The post-contrast scans were obtained at 7,67, 127, 187, 247, and $367 \mathrm{~s}$ after injection of $0.1 \mathrm{mmol} / \mathrm{kg}$ gadopentetate dimeglumine. The images were reformatted in the sagittal and coronal planes with additional maximum intensity projection representation.

\section{ABUS and CAD Systems}

ABUS was performed using the automated breast volume scanner ACUSON S2000 ABUS system (Siemens Medical Solutions, Mountain View, CA, USA) by an experienced radiographer. The radiographer selected the most suitable setting based on the patient's breast size (A-D and DD cups). With the patient in the supine position, anterior-posterior views of the bilateral breasts were initially scanned followed by scanning of the lateral and medial views, which primarily include the outer and the inner breast, in the oblique position. After the image acquisition, the 3D volume data were immediately sent to the workstation and reviewed in multiple orientations (transverse, coronal, and sagittal planes) using multi-planar reconstruction. The scan thickness was displayed at 1-mm intervals without overlap.

In this study, we used OVCAD ${ }^{\text {tm }}$ CAD software (OVCAD system from OView Medical, Inc., Los Altos, CA, USA) to process and display ABUS images on a computer monitor for review with certain areas highlighted or marked for attention. It highlights suspicious regions displayed on an intelligent minimum intensity projection image of the $A B U S$ view.

\section{Image Analysis}

Two specialized breast radiologists with 9 and 11 years of experience, respectively, in academic breast imaging reviewed 40 patients' breast MRIs, and categorized each suspicious lesion by BI-RADS. The CAD-detected lesions that matched suspicious lesions on MRI, were identified and analyzed.

\section{Statistical Analysis}

We statistically analyzed the diagnostic accuracy of CAD, characteristics of CAD-detected lesions and the causes underlying false-positive and false-negative cases, and false readings in general. We calculated the diagnostic performance-sensitivity, specificity, positive predictive value (PPV), and negative predictive value (NPV)-using SAS version 9.2 (SAS Institute, Cary, NC, USA). We then analyzed the characteristics of the CAD-detected lesions using the chi-square test or the Fisher's exact test and calculated the $P$ values, assuming significance at $p$ less than 0.05 .

\section{RESULTS}

The final proportions of malignant and benign lesions in this study were $52.1 \%(49 / 94)$ and $47.9 \%(45 / 94)$, respectively. CAD revealed 62 lesions $(62 / 94,65.9 \%)$ including 35 IDC lesions, 1 mucinous carcinoma, and 1 invasive lobular carcinoma (Table 2). Among the malignant

Table 2. Sensitivity and Specificity of Computer-Aided Detection (CAD) with Automated Breast Ultrasonography for Suspicious Lesions Detected on Breast MRI and CAD-False Lesions

\begin{tabular}{|c|c|c|c|c|}
\hline & \multicolumn{4}{|c|}{ Malignancy } \\
\hline & Benign & Malignancy & Sensitivity (95\%) & Specificity (95\%) \\
\hline Total $(n=94)$ & 45 & 49 & & \\
\hline CAD (-) & 20 & 12 & $75.5(61.1-86.7)$ & $44.4(29.6-60.0)$ \\
\hline \multirow[t]{3}{*}{ CAD (+) } & 25 & 37 & & \\
\hline & \multicolumn{4}{|c|}{ IDC only } \\
\hline & Non IDC & IDC & Sensitivity (95\%) & Specificity (95\%) \\
\hline Total $(n=94)$ & 51 & 43 & & \\
\hline CAD (-) & 24 & 8 & $81.4(66.6-91.6)$ & $47.1(32.9-61.5)$ \\
\hline CAD (+) & 27 & 35 & & \\
\hline
\end{tabular}

IDC = invasive ductal carcinoma

Non IDC including ductal carcinoma in situ, mucinous carcinoma, and invasive lobular carcinoma. 
lesions, we failed to detect 8 IDC lesions, 2 mucinous carcinomas, and 2 DCIS lesions using CAD.

The sensitivity, specificity, PPV, and NPV of CAD for automated breast ultrasound were 75.5\% (37/49), 44.4\% (20/45), 59.7\% (37/62), and 62.5\% (20/32), respectively. CAD facilitated the detection of $81.4 \%$ (35/43) of the invasive ductal cancers and $84.9 \%$ (28/33) of the invasive ductal cancers that showed masses (Table 3 ). CAD also revealed $90.3 \%(28 / 31)$ of the invasive ductal cancers measuring larger than $1 \mathrm{~cm}$ mass (excluding non-masses and lesions smaller than $1 \mathrm{~cm}$ ). Among the $71 \mathrm{MRI}$-detected lesions, 55 were mass lesions on MRI, 15 were non-mass lesions, and 1 was missed. Masses larger than $1 \mathrm{~cm}$ in size were detected at a significantly higher rate $(P<0.05)$, even with invasive ductal cancers that showed masses (Table 4 , Fig. 1).

False-negative lesions included 8 IDC lesions, 2 mucinous carcinomas, and 2 DCIS lesions. The mean sizes of the true-positive versus false-negative mass lesions were $2.08 \pm 0.85 \mathrm{~cm}$ versus $1.6 \pm 1.28 \mathrm{~cm}(P<0.05)$. The falsepositive lesions included a sclerosing adenosis and a case of usual ductal hyperplasia. Additionally, the false CAD marks for pseudo lesions involved 18 marginal or subareolar shadowings, 3 simple cysts, a hematoma, and a skin wart (Fig. 2). Table 4 also shows the correlation between CAD- positive lesions based on MRI and ABUS findings.

\section{DISCUSSION}

HHUS showed promising results as a breast cancer screening tool in the past decade. However, it remains somewhat challenging and time-consuming for radiologists. ABUS may address these limitations by greatly reducing operator dependency and providing high-resolution $3 \mathrm{D}$ images (5). Although ABUS has many advantages over HHUS, it is still associated with a few disadvantages.

In a number of prior studies, the mean diameters of ABUS-detected lesions were larger than those of the handheld-detected lesions, with varying cut-offs $(2,6$, 7). Therefore, it is possible that ABUS reflects the $C A D$ detection sensitivity, especially in size. In this study, the mean sizes of the true-positive versus false-negative mass lesions were $2.08 \pm 0.85 \mathrm{~cm}$ vs. $1.6 \pm 1.28 \mathrm{~cm}(P<0.05)$.

In previous studies, Chang et al. (8) reported statistically significant differences in ABUS detection rates of factors including margin, orientation, and boundary, and van Zelst et al. (9) suggested that a higher BI-RADS category indicated a higher detection rate on ABUS. In our study, the lesion shape, orientation, and margin showed statistically

Table 3. Sensitivity and Specificity of Computer-Aided Detection (CAD) for Breast Cancer According to Mass/Non-Mass Lesions and Size in MRI

\begin{tabular}{|c|c|c|c|c|c|c|c|c|}
\hline & \multicolumn{4}{|c|}{ Malignancy } & \multicolumn{4}{|c|}{ IDC only } \\
\hline & Benign & Malignancy & Sensitivity (95\%) & Specificity (95\%) & Non IDC & IDC & Sensitivity (95\%) & Specificity $(95 \%)$ \\
\hline \multicolumn{9}{|c|}{ Mass in MRI $(n=55)$} \\
\hline CAD (-) & 16 & 8 & $78.4(61.8-90.2)$ & $88.9(65.3-98.6)$ & 19 & 5 & $84.9(68.1-94.9)$ & $86.4(65.1-97.1)$ \\
\hline CAD (+) & 2 & 29 & & & 3 & 28 & & \\
\hline \multicolumn{9}{|c|}{ Non-mass in MRI $(n=15)$} \\
\hline CAD (-) & 4 & 4 & $63.6(30.8-89.1)$ & $100.0(39.8-100.0)$ & 5 & 3 & $66.7(29.9-92.5)$ & $83.3(35.9-99.6)$ \\
\hline CAD (+) & 0 & 7 & & & 1 & 6 & & \\
\hline \multicolumn{9}{|c|}{ Size in MRI: < $1(n=22)$} \\
\hline CAD (-) & 16 & 4 & $20.0(0.5-71.6)$ & $94.1(71.3-99.9)$ & 17 & 3 & $25.0(0.6-80.6)$ & $94.4(72.7-99.9)$ \\
\hline CAD (+) & 1 & 1 & & & 1 & 1 & & \\
\hline \multicolumn{9}{|c|}{ Size in MRI: $\geq 1(n=49)$} \\
\hline CAD (-) & 4 & 8 & $81.8(67.3-91.8)$ & 80.0 (28.4-99.5) & 7 & 5 & $87.2(72.6-95.7)$ & $70.0(34.8-93.3)$ \\
\hline CAD (+) & 1 & 36 & & & 3 & 34 & & \\
\hline
\end{tabular}

IDC = invasive ductal carcinoma

Non-IDC including ductal carcinoma in situ, mucinous carcinoma, and invasive lobular carcinoma

A single missing record involved mass/non-mass in MRI, and the sum was $70(55+15)$ instead of 71 . 
significant differences in $C A D$ detection rate $(P<0.01)$ on ABUS.

ABUS provides volumetric images with many slices and large FOV, which can lead to fatigue and missed radiological findings involving the large FOV, leading to interpretation errors, and ultimately decreasing the accuracy of detection. Skilled and accurate interpretation of $A B U S$ requires considerable radiological training and experience (10). Therefore, a CAD system with ABUS may be more feasible for radiologists in practice. Previous studies have shown positive results supporting the diagnostic utility of CAD with ABUS $(4,11)$. As shown in our study, the sensitivity, specificity, PPV, and NPV of CAD with automated breast ultrasound were $75.5 \%, 44.4 \%, 59.7 \%$, and $62.5 \%$, respectively. The sensitivity of this study was relatively low (75.5\%), because we used the CAD with ABUS for suspicious lesions detected on breast MRI. Breast MRI has proven to be the most sensitive tool for detecting breast carcinoma (12).
Therefore, we enrolled patients with multiple suspicious lesions on preoperative MRI, and included many small and subtle lesions in this study.

Our study analyzed the diagnostic performance of ABUS with CAD for lesions detected using MRI. MR-directed ultrasound, also referred to as second-look ultrasound and targeted ultrasound, has been shown to be useful in identifying lesions detected initially on MRI. In one previous study, the role of MRI was indispensable for preoperative assessment, although the combination of HHUS and ABUS showed the highest sensitivity. In addition, ABUS imaging was better than HHUS for preoperative evaluation (6).

We analyzed MRI lesions that correlated with detection rates on $C A D$ with $A B U S$, and the detection rate was slightly higher if a lesion on MRI was defined as a mass $(31 / 55$, $56.4 \%)$ rather than a non-mass $(7 / 15,46.7 \%)$ lesion. This result suggests the same trend, albeit weaker, as that of a previous study in which the HHUS detection rates were

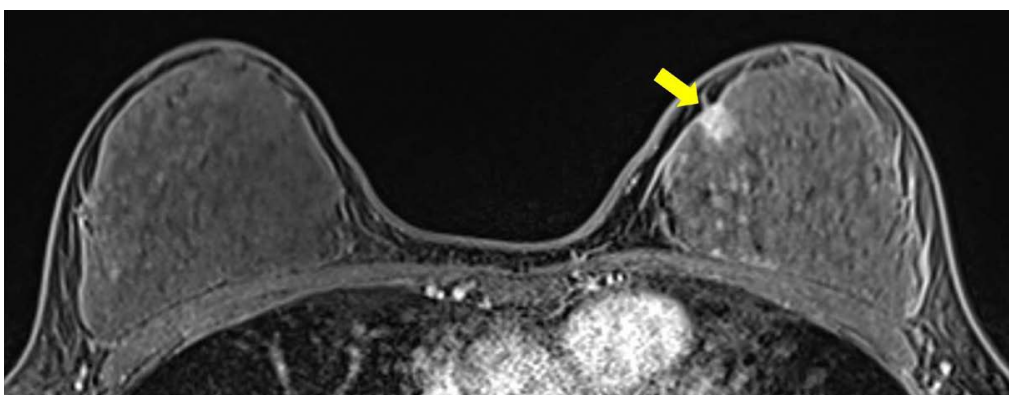

a

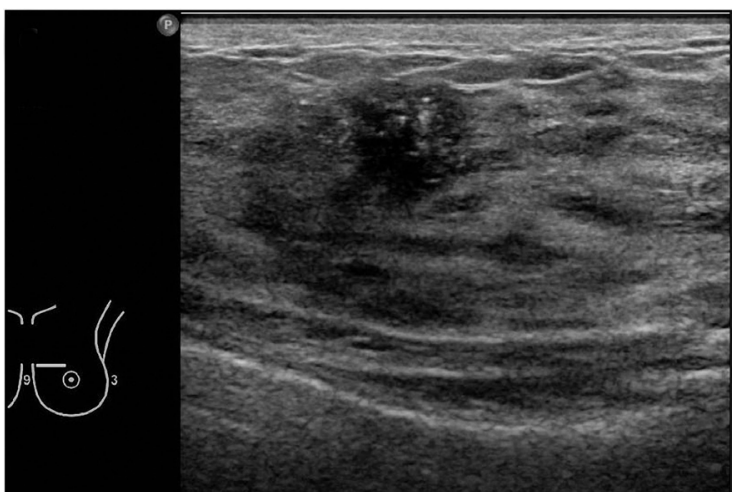

c

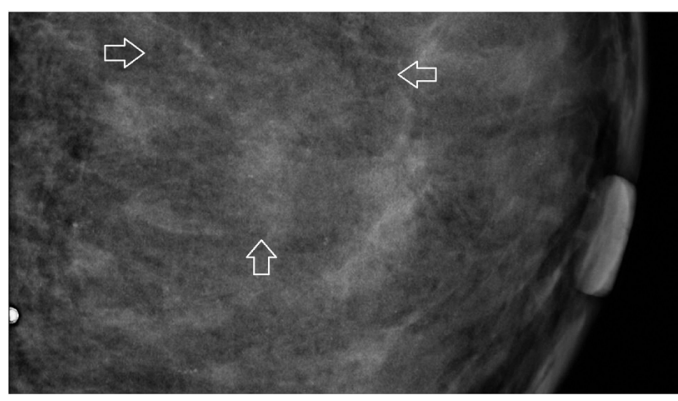

b

Fig. 1. Images from a 32-year-old woman with a suspicious lesion detected on MRI and investigated subsequently with automated breast ultrasound (ABUS). (a) MRI image showed an approximately $1.1 \mathrm{~cm}$ enhancing mass at the 10 o'clock position on the left breast (arrow). (b) Mammography showed microcalcifications with suspicious architectural distortion involving the upper left inner quadrant (arrows). (c) Handheld ultrasonography revealed about 1.1-cm irregular mass with microcalcifications in the same direction. (d) 3D ABUS revealed correlating suspicious lesion on CAD in the left AP and medial views, later confirmed as invasive ductal carcinoma. 
$58 \%$ for mass versus $29 \%$ for non-mass lesions $(13,14)$, and the pathologically invasion by malignant lesions with space-occupying mass formation showed a higher detection rate. In our study, $81.4 \%(35 / 43)$ of the invasive ductal cancer was detected using CAD. CAD was used to detect $84.9 \%(28 / 33)$ of the invasive ductal mass cancers (exclude

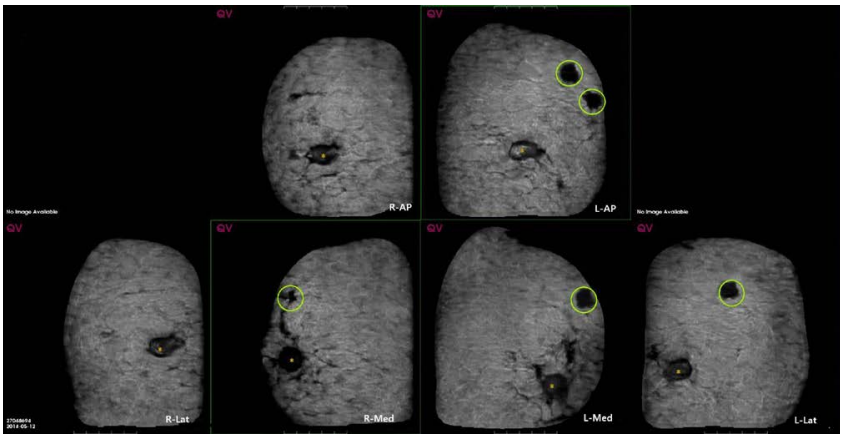

a

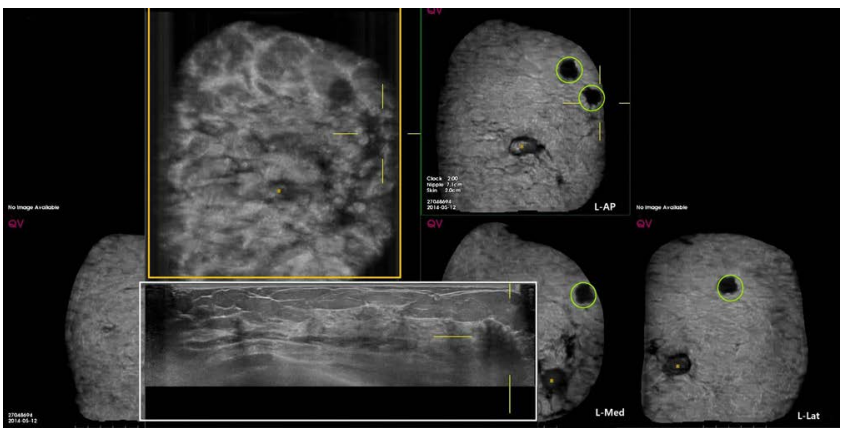

C

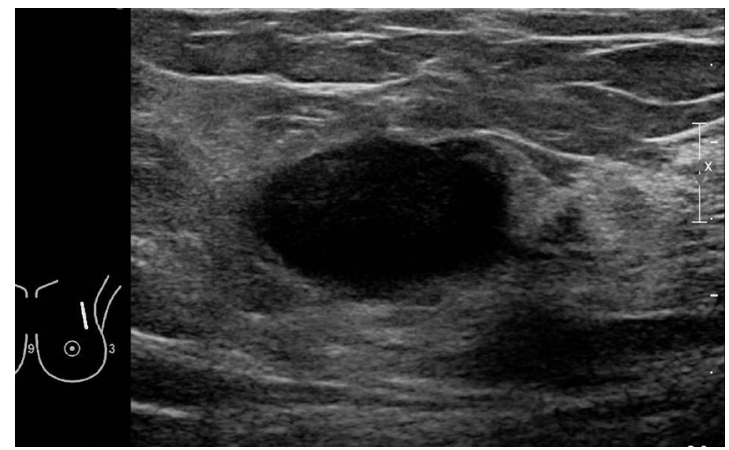

e

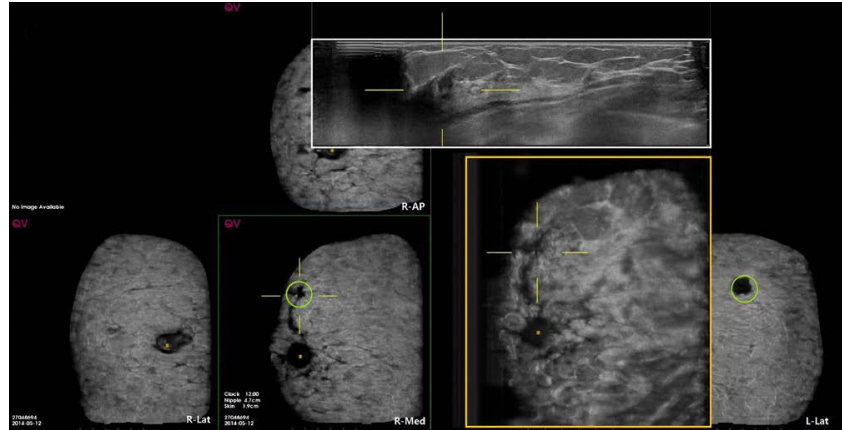

b

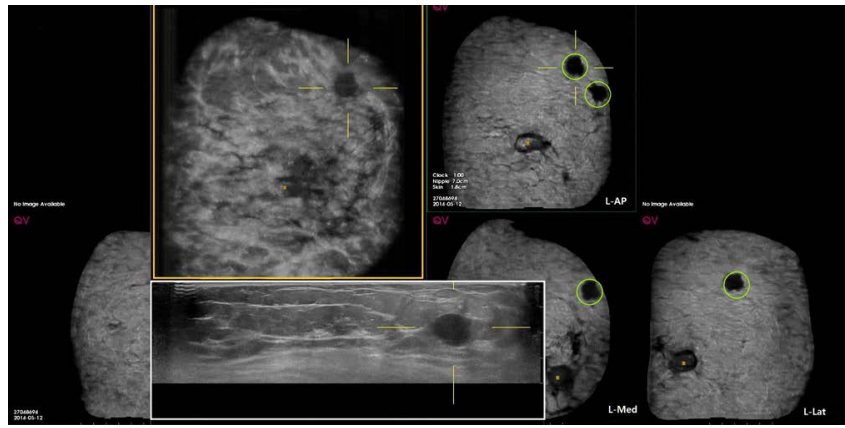

d

Fig. 2. A 62-year-old woman who showed three CAD-detected lesions in both breasts. (a) CAD revealed a suspicious lesion in the right breast and only one marked lesson in the right medial view. The other two marked lesions involved the left breast: one was only marked in the left AP view, and the other one was marked in the whole 3D views. (b) Axial (white box) and maximal intensity projection (MIP) reconstruction image (yellow box) shows a right breast lesion that was confirmed as a pseudo lesion based on marginal shadowing. (c) Axial (white box) and MIP reconstruction image (yellow box) of one of the left breast lesions, which was marked by CAD only on AP view; it was also a pseudo lesion due to marginal shadowing. (d) Axial (white box) and MIP reconstruction image (yellow box) of the other left breast lesion, which was entirely marked by CAD in 3D view, and confirmed as IDC. (e) HHUS image of a biopsy-proven IDC lesion involving left breast shows a 1.8-cm marked hypoechoic mass with microlobulation in the left 2-h direction. (f) MRI of biopsy-proven IDC lesion shows 1.7-cm markedly enhanced mass in the left breast at the 1 o'clock position. 
Table 4. Correlation between Computer-Aided Detection (CAD)-Positive Lesions Based on MRI and Automated Breast Ultrasonography (ABUS)

\begin{tabular}{|c|c|c|c|c|c|}
\hline $\mathrm{N}(\%)$ & Missing & Total & CAD (-) & CAD (+) & P value \\
\hline \multicolumn{6}{|l|}{ Size in MRI } \\
\hline$<1$ & $0(0)$ & $22(31.0)$ & $20(90.9)$ & $2(9.1)$ & $<0.001$ \\
\hline$\geq 1$ & & $49(69.0)$ & $12(24.5)$ & $37(75.5)$ & \\
\hline \multicolumn{6}{|l|}{ Mass/non-mass in MRI } \\
\hline mass & $1(1.4)$ & $55(77.5)$ & $24(43.6)$ & $31(56.4)$ & 0.504 \\
\hline non-mass & & $15(21.1)$ & $8(53.3)$ & $7(46.7)$ & \\
\hline \multicolumn{6}{|l|}{ Mass shape in MRI } \\
\hline oval & $16(22.5)$ & $25(35.2)$ & $15(60.0)$ & $10(40.0)$ & 0.0255 \\
\hline round, irregular & & $30(42.3)$ & $9(30.0)$ & $21(70.0)$ & \\
\hline \multicolumn{6}{|l|}{ Mass margin in MRI } \\
\hline circumscribed & $16(22.5)$ & $17(23.9)$ & $14(82.4)$ & $3(17.6)$ & 0.0001 \\
\hline not circumscribed & & $38(53.5)$ & $10(26.3)$ & $28(73.7)$ & \\
\hline \multicolumn{6}{|l|}{ Internal pattern in MRI } \\
\hline homogeneous & $1(1.4)$ & $20(28.2)$ & $15(75.0)$ & $5(25.0)$ & 0.0019 \\
\hline not homogeneous & & $50(70.4)$ & $17(34.0)$ & $33(66.0)$ & \\
\hline \multicolumn{6}{|l|}{ Non-mass distribution in MRI } \\
\hline not segmental, linear & $56(78.9)$ & $6(8.5)$ & $0(0.0)$ & $6(100.0)$ & 0.0014 \\
\hline segmental, linear & & $9(12.7)$ & $8(88.9)$ & $1(11.1)$ & \\
\hline \multicolumn{6}{|l|}{ Kinetic-initial in MRI } \\
\hline not fast & $1(1.4)$ & $23(32.4)$ & $11(47.8)$ & $12(52.2)$ & 0.804 \\
\hline fast & & $47(66.2)$ & $21(44.7)$ & $26(55.3)$ & \\
\hline \multicolumn{6}{|l|}{ Kinetic-delayed in MRI } \\
\hline not washout & $1(1.4)$ & $34(47.9)$ & $12(35.3)$ & $22(64.7)$ & 0.089 \\
\hline washout & & $36(50.7)$ & $20(55.6)$ & $16(44.4)$ & \\
\hline \multicolumn{6}{|l|}{ Shape in ABUS } \\
\hline oval & $4(5.6)$ & $15(21.1)$ & $13(86.7)$ & $2(13.3)$ & 0.0001 \\
\hline round, irregular & & $52(73.2)$ & $16(30.8)$ & $36(69.2)$ & \\
\hline \multicolumn{6}{|l|}{ Orientation in ABUS } \\
\hline parallel & $4(5.6)$ & $48(67.6)$ & $25(52.1)$ & $23(47.9)$ & 0.0209 \\
\hline not parallel & & $19(26.8)$ & $4(21.1)$ & $15(78.9)$ & \\
\hline \multicolumn{6}{|l|}{ Margin in ABUS } \\
\hline circumscribed & $4(5.6)$ & $16(22.5)$ & 15 (93.8) & $1(6.3)$ & $<0.001$ \\
\hline not circumscribed & & $51(71.8)$ & $14(27.5)$ & $37(72.5)$ & \\
\hline \multicolumn{6}{|l|}{ Echo pattern in ABUS } \\
\hline an, iso, hyper & $4(5.6)$ & 7 (9.9) & $5(71.4)$ & $2(28.6)$ & 0.2251 \\
\hline hypo, heterogenous, complex & & $60(84.5)$ & $24(40.0)$ & $36(60.0)$ & \\
\hline \multicolumn{6}{|l|}{ Posterior feature in ABUS } \\
\hline no, enhance & $4(5.6)$ & $55(77.5)$ & $26(47.3)$ & $29(52.7)$ & 0.1583 \\
\hline shadow, complex & & $12(16.9)$ & $3(25.0)$ & $9(75.0)$ & \\
\hline
\end{tabular}


non-mass). We found a statistically significant correlation between CAD with ABUS detection rate and the size of the lesions characterized as masses on MRI. Masses measuring greater than $1 \mathrm{~cm}$ in size were more likely to be detected on $C A D$, although we did not find this association between size and the likelihood of non-mass lesions detected on MRI. The discrepancies between mass and non-mass lesions may be because mass lesions, defined as three-dimensional space-occupying lesions, are more readily defined with $C A D$ on ABUS.

In this study, we found 23 false CAD marks. The false CAD marks for pseudo lesions were easily identified as benign rather than suspicious. The false readings on $C A D$ were attributed to typical marginal or subareolar shadowing, simple cysts, a cystic hematoma, and a skin wart, not mimicking a true breast lesion.

Our study has a few limitations. First, the study comprised a relatively small sample that lacked representativeness. Second, in some cases biopsy was not confirmed, and this might have affected outcomes. Third, two radiologists independently reviewed the MRI and HHUS and MRI and ABUS; however, this study did not provide the radiologists' performance analysis such as inter-observer agreement. In this study, we focused on the diagnostic performance of $C A D$ with $A B U S$ and interpreted the lesions associated with errors in the CAD system. The last limitation of this study is that we were not always confident about the precise MRIultrasound correlations. However, we stipulated stringent criteria to determine the sonographic correlation, such as lesion position, size, and similarity in shape. As such, we can guarantee true MRI-ultrasound correlations.

In this study, we determined the diagnostic performance of $C A D$ with $A B U S$ for suspicious lesions detected on breast $M R I$, the characteristics of CAD-detected lesions, and the causes of false positives, false negatives, and false $C A D$ marks. In conclusion, it was not difficult for operators familiar with CAD combined with ABUS to identify false $C A D$ marks for pseudo lesions. We demonstrated that $C A D$ with ABUS showed a reliable diagnostic performance for invasive ductal cancers involving masses larger than $1 \mathrm{~cm}$.

\section{Acknowledgments}

The breast MRI study group was financially supported by the Korean Society of Magnetic Resonance in Medicine.

\section{REFERENCES}

1. An YY, Kim SH, Kang BJ. The image quality and lesion characterization of breast using automated whole-breast ultrasound: a comparison with handheld ultrasound. Eur J Radiol 2015;84:1232-1235

2. Jeh SK, Kim SH, Choi JJ, et al. Comparison of automated breast ultrasonography to handheld ultrasonography in detecting and diagnosing breast lesions. Acta Radiol 2016;57:162-169

3. Wang ZL, Xu JH, Li JL, Huang Y, Tang J. Comparison of automated breast volume scanning to hand-held ultrasound and mammography. Radiol Med 2012;117:12871293

4. van Zelst JCM, Tan T, Platel B, et al. Improved cancer detection in automated breast ultrasound by radiologists using computer aided detection. Eur J Radiol 2017;89:5459

5. Shin HJ, Kim HH, Cha JH. Current status of automated breast ultrasonography. Ultrasonography 2015;34:165-172

6. Kim Y, Kang BJ, Kim SH, Lee EJ. Comparison and combination of two ultrasound modalities, handheld ultrasound and automated breast volume scanner, with and without knowledge of MRI. Iran J Radiol 2018;15:e60176

7. Wang HY, Jiang YX, Zhu QL, et al. Differentiation of benign and malignant breast lesions: a comparison between automatically generated breast volume scans and handheld ultrasound examinations. Eur J Radiol 2012;81:3190-3200

8. Chang JM, Moon WK, Cho N, Park JS, Kim SJ. Radiologists' performance in the detection of benign and malignant masses with 3D automated breast ultrasound (ABUS). Eur J Radiol 2011;78:99-103

9. Van Zelst JC, Platel B, Karssemeijer N, Mann RM. Multiplanar reconstructions of 3D automated breast ultrasound improve lesion differentiation by radiologists. Acad Radiol 2015;22:1489-1496

10. Kim JH, Cha JH, Kim N, et al. Computer-aided detection system for masses in automated whole breast ultrasonography: development and evaluation of the effectiveness. Ultrasonography 2014;33:105-115

11. Drukker K, Sennett CA, Giger ML. Automated method for improving system performance of computer-aided diagnosis in breast ultrasound. IEEE Trans Med Imaging 2009;28:122-128

12. Berg WA, Gutierrez L, NessAiver MS, et al. Diagnostic accuracy of mammography, clinical examination, US, and MR imaging in preoperative assessment of breast cancer. Radiology 2004;233:830-849

13. Wiratkapun C, Duke D, Nordmann AS, et al. Indeterminate or suspicious breast lesions detected initially with MR 
imaging: value of MRI-directed breast ultrasound. Acad Radiol 2008;15:618-625

14. Abe H, Schmidt RA, Shah RN, et al. MR-directed ("Second-
Look") ultrasound examination for breast lesions detected initially on MRI: MR and sonographic findings. AJR Am J Roentgenol 2010;194:370-377 